Editorial

\section{Immunometabolism-A Field Rapidly Gaining International Recognition and Reputation}

Immunometabolism is a rapidly expanding field of investigation linking the disciplines of immunology and metabolism. The late Eric A. Newsholme, based in the University of Oxford, was a pioneer in the field, publishing several key papers on lymphocyte and macrophage metabolism in the early to mid-1980s [1]. Increasing interest in this area over the last decade may be linked to the obesity epidemic as it has become clear that obesity impacts the immune system and promotes inflammation, and subsequent inflammation contributes to the pathology of chronic conditions and diseases such as diabetes and cardiovascular disease. Interactions between key common (or novel) metabolites and immune cell function such as activation or inhibition of signaling pathways, expression of receptors and adhesion molecules, regulation of gene expression, activation and proliferation of specific sub-sets of lymphocytes or phagocytic cells may offer substantial therapeutic promise. It is worth quoting Benjamin Franklin the American inventor, statesman and publisher, who under the pseudonym Poor Richard wrote in his Almanac in 1737, "To lengthen thy life, lessen thy meals" [2] so highlighting the fact that connections between diet and health have long been appreciated. We aim in this new journal to publish articles that describe molecular processes that impact health by changing metabolism and immune function. Several relevant research topics are described below.

Nutritional imbalance is a major issue in the world-wide human population as overnutrition and undernutrition are significant public health issues. In many mammalian species, excess nutrients are converted to lipids and stored in various adipose tissue sites. During fasting and starvation, stored nutrients are metabolized to provide energy and heat to maintain vital reproductive and physiological processes.

Adipose tissue mass changes in response to lipid storage or release. This influences the secretion of hormones and cytokines from adipose tissue (adipocytokines). Adipocytokines have important signaling functions which can influence immune cell proliferation and immune responses. An example is Tumor Necrosis Factor- $\alpha$ (TNF- $\alpha$ ) which can be secreted from adipose tissue. It is released at high levels during obesity but decreased after weight loss. TNF$a$ is a pro-inflammatory cytokine and is known to be essential for the development of insulin resistance in high-fat diet-induced obesity in animal models and indeed in obese humans. TNF-a can be secreted from both adipose tissue-localized macrophages and from adipocytes. Interleukin 6 (IL-6) and Leptin are other adipocytokines that are additionally released at higher levels in obesity, which impact directly and indirectly on immune cell function. of Creative Commons Attribution 4.0 International License. 
Several types of adipose tissue residing immune cells are affected by changes in adipocytokine levels. Macrophages comprise more than 50\% of adipose tissue-resident immune cells. During obesity, additional macrophages are recruited into the adipose tissue in response to various stimulatory factors released from the adipose tissue. The macrophages are subsequently polarized into pro-inflammatory activated macrophages (previously termed M1 macrophages) in response to Interferon- $\gamma$ (IFN- $\gamma$ ), which is secreted by effector $\mathrm{T}$ cells and other immune cells in the adipose tissue, stimulating macrophages to release TNF- $\alpha$ in addition to other inflammatory molecules including IL-1 $\beta$, IL-6, and IL-12.

Several lymphocyte populations are also located in the adipose tissue. B lymphocytes (B cells) accumulate in the adipose tissue during obesity. Although B cells are best known for the production of antibodies, they also release inflammatory cytokines, such as IL-2 and IL-12, which influence T cell differentiation into Thelper cell type 1 (Th1) versus Th2 cells.

$\mathrm{T}$ lymphocytes ( $\mathrm{T}$ cells) represent the most abundant lymphocyte population in the adipose tissue. Both $\mathrm{CD} 4^{+}$and $\mathrm{CD} 8^{+} \mathrm{T}$ cells are located in the adipose tissue. $T$ cell populations are altered during obesity so favouring the pro-inflammatory state: both $\mathrm{CD} 8^{+} \mathrm{T}$ cells and pro-inflammatory $\mathrm{CD} 4^{+} \mathrm{Th} 1$ cells are elevated, they release the pro-inflammatory cytokine IFN- $\gamma$, while effector $\mathrm{CD} 4^{+}$Th17 cells release the pro-inflammatory cytokine IL-17.

In addition to obesity and overnutrition contributing to chronic inflammation as described above, immune cell metabolism can be changed in response to nutrient or hormone or inflammatory factor stimulation. The major metabolic changes involve a switch to glycolytic metabolism in activated lymphocytes and macrophages, while reducing their pathways of oxidative metabolism. Partial TCA cycle activity may be operational under such conditions but serves to generate metabolic signals. This area of research is now receiving considerable attention due to potential for discovery of novel therapeutic targets.

To recognize the immunometabolism research, I am pleased to announce the launch of the journal Immunometabolism. Immunometabolism will be published online in an open access form. We aim in this journal to centrally reflect the rapid progress that has been made and is being made in this field. Immunometabolism is supported by a formidable editorial board of outstanding scientists and colleagues across the world. Authors will get support from the editors in each step of peer review and subsequent publication.

I look forward to receiving your submissions and welcome your suggestions on how to make this journal a leader in field. 


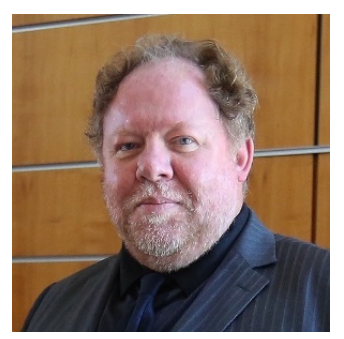

Philip Newsholme

Founding Editor-in-Chief of Immunometabolism School of Pharmacy and Biomedical Sciences, Curtin University, GPO Box U1987, Perth, Australia E-Mail: Philip.Newsholme@curtin.edu.au

\section{REFERENCES}

1. Curi R, de Siqueira Mendes R, de Campos Crispin LA, Norata GD, Sampaio SC, Newsholme P. A past and present overview of macrophage metabolism and functional outcomes. Clin Sci (Lond). 2017 Jun 15; 131(12):1329-1342. doi: 10.1042/CS20170220.

2. Franklin B. Almanac. Philadelphia: The New Printing Office near the market; 1739.

How to cite this article:

Newsholme P. Immunometabolism—A Field Rapidly Gaining International Recognition and Reputation. Immunometabolism. 2019;1:e190001. https://doi.org/10.20900/immunometab20190001 